\title{
American Journal of Clinical Dermatology Supplement
}

\section{The Jungle on the Skin: Cutaneous Microbiome}

\author{
Guest Editors \\ Brigitte Dréno \\ Department of Dermatology, \\ Nantes University Hospital, \\ Nantes, France \\ brigitte.dreno@atlanmed.fr \\ Gil Yosipovitch \\ Miami Itch Center, \\ Dr Phillip Frost Department of Dermatology and Cutaneous Surgery, \\ Miller School of Medicine, University of Miami, \\ Miami, FL, USA \\ gyosipovitch@med.miami.edu
}

Disclosures: This supplement was wholly funded by Laboratoires Dermatologiques Avène-Pierre Fabre Dermo-Cosmétique. Medical writing assistance was also funded by Laboratoires Dermatologiques Avène-Pierre Fabre Dermo-Cosmétique.

Guest Editor Conflicts of Interest: Gil Yosipovitch: consultant and scientific advisory board member of Trevi, Pfizer, Galderma, Novartis, Sanofi Regeneron, Pierre Fabre, and LEO, and research sponsored by Pfizer, Sun Pharma, Novartis, LEO, and Sanofi Regeneron. Brigitte Dréno: advisory board member and/or main investigator for clinical trials for Pierre Fabre, La Roche Posay, Bioderma, Galderma, Sanofi, BMS, Roche, AbbVie, Almirall. 